Research Article

Open Access

\title{
The Influence of Cognitive Behavioral group Interventions for HIV Seropositive Women living in the face of Childhood Sexual Abuse
}

\author{
Eula W Pines ${ }^{\star}$
}

Professor, Troy University School of Nursing, Alabama, Phenix City Campus, USA

\section{Article Info \\ *Corresponding author: \\ Eula W Pines \\ Troy University School of Nursing \\ Alabama, Phenix City Campus \\ USA \\ E-mail: epines@troy.edu}

Received: June 19, 2018

Accepted: June 23, 2018

Published: June 29, 2018

Citation: Pines EW. The Influence of Cognitive Behavioral Group Interventions for HIV Seropositive Women Living in the Face of Childhood Sexual Abuse. Madridge J AIDS. 2018; 2(1): 42-49.

doi: 10.18689/mja-1000108

Copyright: @ $₫ 2018$ The Author(s). This work is licensed under a Creative Commons Attribution 4.0 International License, which permits unrestricted use, distribution, and reproduction in any medium, provided the original work is properly cited.

Published by Madridge Publishers

\begin{abstract}
Purpose

This pilot study investigated the efficacy of cognitive group interventions in reducing traumatic stress and human immunodeficiency virus (HIV) transmission behaviors in HIV seropositive women survivors of childhood sexual abuse.
\end{abstract}

\section{Method}

A quantitative quasi-experimental one-group design was used to test the interventions, which consisted of eight weekly 90-minute group sessions. Baseline data were collected after receiving Institutional Review Board approval. Participants were eleven self- reported HIV positive women survivors with childhood sexual abuse histories. Demographic questions in the questionnaire assessed age, race, ethnicity, and educational level. Two questions measuring engagement in unprotected sex and substance use were asked. Participants completed the 15-question Impact of Event Scale.

\section{Results}

The results showed a clinical significant reduction in the impact-of-event scores and minimal effect on HIV transmission behaviors from pre-test to post-test.

\section{Conclusion}

This pilot study supported the use cognitive behavioral group interventions in reducing traumatic stress associated with childhood sexual abuse. However, the complexity of traumatic stress as a driver of HIV transmission needs further investigation.

Keywords: Cognitive behavioral therapy; Childhood sexual abuse; Traumatic stress; HIV transmission.

\section{Introduction}

Worldwide, 18.8 million women and girls are living in the face of human immunodeficiency virus infection [1] and approximately 120 million girls have experienced childhood sexual abuse (CSA) before age 18 years [1-4]. Childhood sexual abuse is a form of gender-based violence fueling the HIV epidemic in developing countries [1,2] and in the United States [3-11]. HIV comingled with CSA threatens the health and well-being of women. Strong evidence suggests that the resultant traumatic stress provides direct and indirect pathways to high-risk sexual activities including multiple partners, unprotected intercourse, prostitution, sexual acting out, and drives the HIV epidemic [12-14]. Consequently, women experience compromised sense of self and ability to embrace sexuality in healthy and adaptive ways, self-management of HIV 
infection, and poor quality of life [12-14]. Evidence based women focused interventions are needed to reduce traumatic stress and sexual risk behaviors in seropositive women living with CSA and HIV. The aim of this study is to test women focused interventions to mitigate these enduring traumatic stressors in HIV seropositive women with CSA histories. Lazarus and Folkman's transactional model of stress and coping guided this study [14].

\section{Transactional Model of Stress and Coping}

Lazarus and Folkman [14] postulated that the transactions between individuals and their environments are appraised by the individuals as exceeding personal resources and endangering a person's well-being. Consequently, two types of coping may emerge: (1) problem-focused coping, which manages or alters the problem causing the stress, and (2) emotion-focused coping, which regulates emotional responses. Problem-focused coping is appropriate when situations are appraised as amenable, such as choices about relationships [14]. Conversely, emotion-focused coping is appropriate when dealing with situations appraised as not changeable [14], such as HIV infection and a history of CSA [12].

Researchers have found through randomized controlled trials (RCTs) that emotion-focused coping and problemfocused interventions enabled people diagnosed with HIV infection and histories of CSA to express emotions [11]. From this perspective, problem-focused skills were needed to help women manage issues in relationships appraised as amendable, whereas emotion-focused skills were needed to help women cope with situations appraised as not amendable $[11,14]$. Seen from this perspective, cognitive processing for women survivors of CSA and HIV enables women to address feelings of anger, betrayal, shame, guilt, humiliation, anxiety, and confusion by modifying cognitive schema conflicts [4-8, 15-16]. Women's cognitive appraisal of these demands and coping resources influence health outcomes.

The National Institute of Mental Health [17] reported that HIV positive survivors of CSA are less likely to engage in risky sexual behavior if they participate in group interventions. To reduce traumatic stress and sexual risk behaviors, many researchers have included both men and women in the same cognitive group intervention studies [9-14]. However, empowering women survivors of CSA and HIV require targeted women focused group interventions. Few studies have included women only, thus, affecting generalizability of the outcomes. This study addressed this gap in knowledge. The aim of this study is to examine the influence of cognitive behavior group interventions for HIV seropositive women with CSA histories. The inquiry question is: In HIV seropositive women with childhood sexual abuse, will cognitive behavioral group interventions reduce traumatic stress and HIV risky behaviors?

\section{Methodology of Search Process}

Databases search included relevant systematic reviews, meta-analysis, and single randomized controlled trials in the Cochrane Database of Systematic Review, EMbase, PubMed,
CINAHL, Johanna Briggs Institute, Web of Science, and relevant online journals between 2004 and 2017. The keywords included: behavioral interventions and HIV, HIV-seropositive women, childhood sexual abuse, traumatic stress and metaanalysis, group therapy, cognitive behavioral therapy and randomized control trials, stress and coping.

The synthesis of the evidenced literature review are organized into four categories: (1) behavioral interventions and risky sexual behaviors; (2) CBT group interventions and transactional stress model; (3) gender-specific CBT interventions; and (4) and group interventions with men and women.

\section{Literature Review}

\section{Behavioral Interventions and Risky Sexual Behaviors}

Crepaz et al's [18] meta-analysis investigated the efficacy of behavioral interventions to decrease sexual risky behaviors among African-American females in a northern, urban area of the United States. The systematic review covered studies published from January 1988 to June 2007, yielding 37 relevant studies. The researchers used mixed-effect models and a meta-regression statistical model for analysis. Increased efficacy was noted in studies that specifically targeted AfricanAmerican women when researchers used gender and culturespecific materials; groups were facilitated by women and addressed empowerment issues; provided skills training in condom use and role-playing; and taught negotiation skills for safer sex [18].

Carvalho et al's [19] systematic review investigated the effectiveness of behavioral interventions in promoting condom use among HIV- positive women. Studies were included in the analysis if researchers conducted a randomized control trial (RCT) that examined the effects of behavioral interventions on condom use among HIV-positive women; considered at least one HIV-related behavioral outcome; and one follow-up assessment three months after the intervention. They used random effects models to summarize odds ratios that compared intervention and control groups with respect to a dichotomous outcome (consistent versus inconsistent condom use). The review included five primary studies with positive women $(n-725)$. The results of the meta-analysis showed that, compared to care or minimal HIV support interventions, behavioral interventions had no effect on increasing condom use [19].

\section{Gender-Specific CBT Interventions}

Wyatt et al. [7] conducted an (RCT) to test the efficacy of a gender-specific, culturally congruent, enhanced sexual health intervention (ESHI). The ESHI guided CBT approaches designed to enhance medication adherence and risk reduction behaviors. The participants included HIV-positive, AfricanAmerican and European-American women with histories of CSA ( $n=127)$.

In the study, baseline assessment was obtained and all women participants were randomly assigned either to the ESHI or the attention control with repeated measures and time-lagged design. This design was similar to those of casemanagement approaches. The findings provided support for 
culturally congruent CBT psycho education, prevention, and intervention for HIV-positive women with histories of CSA and medical non-adherence issues. Interventions were clearly described, but participants' treatment and control process were unclear [7].

Sikkema et al. [9] investigated outcomes of a 15-session CBT-group intervention with HIV-positive men and women targeted at CSA and HIV risky behaviors. Group participants included women $(n=107)$ and men $(n=90)$ with histories of CSA. The participants were randomly assigned to one of three groups: a 15-session support group intervention, a 15-session comparison support group, and a waitlist control group. Traumatic stress symptoms were assessed at baseline and post-intervention. Analyses conducted on the three-condition comparisons, followed by the two-condition comparison between the coping and support groups [9].

The intervention model integrated the cognitive theory of stress and coping with CBT techniques and was effective for treating victims of sexual trauma [14]. The coping framework of Lazarus and Folkman [14] was employed to demonstrate appraisals of stressors related to HIV and sexual trauma. Stress associated with traumatic events was measured by the Impact of Events Scale (IES) [20]. A comparison group was established in parallel to a standard therapeutic HIV support group to provide a supportive environment for participants addressing the effects of HIV and trauma. The patients were randomly assigned to one of the three groups.

The findings revealed that participants in the coping group intervention demonstrated reductions in intrusive traumatic symptoms as compared with the support group condition [9]. No differences were found between the support group intervention and the waitlist condition. Researchers concluded that the test of significance documented the meaningfulness of change in traumatic stress symptoms.

\section{Group Interventions and CBT Theory}

Wong et al. [21] investigated the effects of behavioral interventions on substance abuse among people diagnosed with HIV. In the RCT, participants included men and women between the ages of 18 and 55 years who were diagnosed with HIV. The researchers adapted components of the Healthy Living Project (HLP). The HLP is a Center for Disease Control and Prevention evidence-based, behavioral, face-to-face, 15-session, 90-minute, and case-manager (CM) intervention for HIV-positive individuals. The HLP is based on CBT principles and the social cognitive theory. During the study, the case managers presented participants with three modules: (1) stress, coping, and adjustment; (2) safe sex behaviors; and (3) health behaviors (CDC, 2010) [22]. The HLP targets heterosexual women and men, gay men, and injection drug users. The researchers found a significant reduction in the use of drugs such as alcohol and marijuana, and, over time, noted a significant reduction in the use of heroin, cocaine, crack, and speedball.

The findings suggested that the integration of the $\mathrm{CM}$ intervention model delivered individually may result in significant and sustained reductions in substance use among HIV-positive men and women [21]. Based upon these findings, the integration of the CM approach before, during, and after CBT group interventions provided women with additional support.

\section{Integrating CBT Group Interventions Transactional Stress Theory}

Chesney et al. [23] an RCT to compare the effects of a theory-based coping effectiveness training intervention (CET) with an active informational control HIV-information condition and waiting list control condition on psychological distress and positive mood in HIV-seropositive gay men. Participants included self-identified gay ( $\mathrm{n}-149)$ or bisexual men between the ages 21 and 60 who reportedly depressed feelings with CD4 levels of 200-700 cells $/ \mathrm{mm}^{3}$. The CET and HIV-information participants attended ten 90-minute sessions during the three-month intervention phase and six maintenance sessions over the remainder of the year [23].

Participants were assessed at baseline and at three, six, and twelve months. After the three-month intervention phase, when compared with HIV-info, CET participants showed significantly decreases in perceived stress and burnout, and regression analyses indicated significant increases in coping self-efficacy mediated the improvements in perceived stress and burnout. Compared to wait control list (WCL), CET participants also showed significant decreased anxiety, and regression analyses indicated increased positive states of mind mediated improvement in anxiety. Moreover, treatment differences for positive morale were maintained at 6 and 12 months. Optimism continued to increase in the CET and HIVinfo treatment groups during the maintenance phase. The evidence suggests that CET can be an effective strategy for managing psychological distress and improving positive psychological states in patients confronting chronic illness such as HIV and CSA [23].

Meade et al. [4] investigated the effects of integrating the cognitive theory of stress and cognitive behavioral strategies within a transactional framework for understanding CSA outcomes in a CBT coping skills group. Participants were randomly assigned to the experimental coping group or a timematched comparison support group. Interventions were delivered in a group format over 15 weekly, 90 -minute sessions. A diverse sample of HIV-positive men and women $[n=247$ with histories of CSA was recruited from HIV/AIDS organizations.

The researchers found that many HIV-positive clients (primarily with CSA histories) continued to exhibit substance abuse behaviors [4]. However, group interventions addressing HIV and sexual trauma were more effective in reducing alcohol and cocaine use; and the effects were sustained at a 12-month follow-up. Meade et al. [4] asserted that integrating mental health treatment into HIV prevention may improve outcomes.

\section{CBT Group Interventions with HIV Positive Women with CSA}

Puffer et al. [24] conducted a cognitive behavior group intervention with HIV-positive women in an RCT, the aims of which were to reduce traumatic stress and sexual-risk behaviors among ethnically diverse women ranging from 26 to 57 years of age $(n=52)$. The participants were primarily HIV-positive African- 
Americans with histories of CSA and substance abuse. Participants' demographics and socioeconomic statuses mirrored the clients in this study. The researchers focused on clinical issues that emerged among female participants who received the intervention. The researchers' direct clinical observation revealed the importance of supporting women in recognizing the connections between trauma, psychological distress, and high-risk behaviors. In addition, the women successfully applied psycho educational materials, expressed an increased sense of power over their relationships and behaviors, and developed more adaptive cognitive behavioral skills [24].

During the trial, attrition and attendance data were collected. The participants completed two qualitative, freeresponse questionnaires: a participant attendance questionnaire designed to assess factors related to high or low levels of attendance, and an expectations questionnaire to assess initial expectations of the intervention and ways in which expectations were met or not met [24]. In addition, clinicians wrote clinical process notes after each session about the participants' responses to session content and interactions among group members and clinicians. The clinicians administered four follow-up assessments to measure changes in psychological and behavioral variables at different time points after the intervention.

The findings revealed that group intervention reduced traumatic stress and sexual-risk behaviors among participants. Puffer et al. [24] noted through direct clinical observation that recognizing connections among trauma, psychological distress, and high-risk behaviors was a powerful and new experience for the women.

The science investigating the efficacy of CSA and HIV interventions in women was limited. The inquiry question is: In adult HIV seropositive women with histories of childhood sexual abuse, does using cognitive behavioral therapy group interventions targeted at childhood sexual abuse (CSA) and HIVrisky sexual behaviors reduce traumatic stress and HIV transmission behaviors?

\section{Methodology \\ Design}

A quasi-experimental one-group pre-test and post-test intervention design was used in this clinical project. Eleven women volunteered to participate in the CBT group interventions. Participants were given the 15-questionlmpact of Event Scale (IES) [20] which asked participants to respond to questions about past traumatic events, and the principal investigator's demographic survey was composed of six questions on participants' age, race, ethnicity, and education level. It also included two questions about unprotected sex and drug use during the past month. The IES and demographic questionnaire were administered pre-and post-intervention.

\section{Sample}

A convenience sample of 11 women volunteered to participate in the study. The participants were between the ages 24 and 59 years and able to read and speak English at the $8^{\text {th }}$ grade level. The participants self-identified that they have a history of CSA before age 18 years. Participants in the study were excluded if they were diagnosed with psychotic disorders according to clinical records.

All instruments and study protocol were approved through the University's Institutional Review Board. Written informed consents were received from all participants.

The sample of participants was recruited from women attending weekly HIV support group at the HIV service organization. Prior to the intervention, each participant completed a 15-question Impact of Event Scale (IES) [20] asking for their response to questions about past traumatic events, and the principal investigator's demographic survey, which included four questions on participants' age, race and ethnicity, and education level and two questions measuring engagement in unprotected sex and substance use in the past month.

Participants attended eight weekly 90-minute group sessions at which activities included meditations, group discussions, role play, journaling, and relaxation exercises. Topics included identifying maladaptive coping behaviors associated with traumatic events, differentiating between emotion-focused coping and problem-focused coping behaviors, supportive networks and healthy relationships, safe sex, effective communication skills, and negotiation skills. Topics were adapted from Living in The Face of Trauma [6].

\section{Data Collection Plan}

The IES [20] was used to assess participants' subjective responses to traumatic life events. Corcoran and Fischer [25] found that the subscales of IES's internal consistency were appropriate and based on two separate samples. The coefficients ranged from 0.79 to 0.92 . The average of 0.86 for intrusive subscale and 0.90 for avoidance subjects with stress response symptoms on the 15-item IES gave a mean total stress score of 39 . The mean avoidance subscale score (items $2,3,7,8,9,12,13$, and 15 ) was 18.2 ( $S D=10.8$, range 0-38). Thus, the IES has been assessed as reliable [25].

The IES is sensitive to change, specifically for detecting changes in clinical status over time, and particularly in detecting the differences in responses to traumatic events of varying severity [25]. Corcoran and Fischer [25] noted significant changes in the IES subscale scores of outpatient subjects receiving bereavement treatment over the course of time. From this perspective, the IES demonstrates validity and is an appropriate instrument to measure the impact of traumatic stress on HIV-positive women with histories of CSA.

The IES consists of 15 items, seven of which measure symptoms such as intrusive thoughts, nightmares, intrusive feelings, and imagery. The instrument "taps" avoidance symptoms such as numbness of response and avoidance of feelings, situations, and ideas. The scores are totaled to provide a subjective score [20]. The instrument can be used for repeated measurements over a period of time. The instrument is sensitive to change and is useful for monitoring individuals' progress after group intervention [25]. Thus, the instrument is valid and reliable [25]. 
Horowitz et al. [20] indicated that all items of the IES are anchored to a specific stressor. Respondents are asked to rate the items on a four-point scale according to how often each has occurred in the past seven days. Researchers have the option to modify the time of past experiences [20]. For example, this author modified the participants' time of the past experience to read "during the past month." The four points on the scale are 0 (not at all), 1 (rarely), 3(sometimes), and 5 (often). Higher scores indicate greater distress, with scores of 36 and over representing significant distress [26,27]. Other measures included the researcher-designed demographic questionnaire and the participant survey.

\section{Demographic Questionnaire}

A questionnaire to measure demographic characteristics of the sample included age, race, ethnicity, and educational level, and two yes-or-no questions measuring engagement in unprotected sex and substance use: (a) "In the past month, I have used condoms during each sexual activity" and (b) "In the past month, I have used street drugs to cope." The participants completed the questionnaire at pre-and postintervention data collection times.

\section{Data Analysis}

Descriptive statistics were used to describe the sample. The Wilcoxon t-tests were used to assess differences in preand post-intervention IES scores. Results of the findings are presented. A confidence interval of $95 \%$ was used with alpha set at 0.05 [28].

\section{Descriptive Statistics for Participant Demographics}

The participants were females. The participants' ethnicities were reported as follows: 1 (10.0\%) American Indian/Alaskan Native, 5 (50.0\%) Black/African American, 2 (20.0\%) Hispanic/Latino, 1 (10.0\%) Multi-Racial and 1 (10.0\%) white. One individual did not indicate ethnicity. The average participant was 49.55 (10.62) years of age and had 12.00 (1.63) years of education. One individual did not indicate years of education. The descriptive statistics for the participants' continuous and discreet demographic variables are listed in tables 1 and 2, respectively.

\section{Descriptive Statistics for Condom Use \& Street Drug Use}

The participants willingly answered two questions pertaining to sexual behavior and drug use over the past month. The descriptive statistics for these items are listed in table 3. Approximately half $(n=6,54.5 \%)$ of the respondents indicated they had not used condoms during sexual activity in the past month. However, it was not possible to assess whether they had sexual activity in the past month. Therefore, the lack of condom use did not necessarily indicate a lack of protection during sexual behavior. A large majority $(n=10$, 90.9\%) of the respondents indicated they had not used street drugs to cope within the past month.

\section{The Impact of Event Scale}

The descriptive statistics for the participants' responses at pre-test and post-test are listed in Tables 4 and 5, respectively. The respondents were also asked to respond to questions on the 15-item Impact of Event Scale [20]. The distributions of participants' overall scores at pre-test and post-test are displayed in figures 1 and 2, respectively. The pre-test scores demonstrated a slight negative skew; however, the distributions were not a conclusive test of normality given the small sample size. The descriptive statistics and paired t-test statistics are listed in tables 7 and 8 , respectively. The test revealed a clinical significant decrease in event impact scores from pre-test $(M=1.33, S D=0.40)$ to post-test $(M=0.91, S D=0.27), t(10)=3.72, p=.004$. This suggests that the intervention had a positive impact on the participants' thoughts as it related to their traumatic experiences.

Table 1. Descriptive Statistics for Sample Categorical Demographics

\begin{tabular}{lcc}
\hline Variable & $\mathrm{N}$ & $\%$ \\
\hline Gender & 11 & 100.0 \\
Female & 0 & 0.0 \\
Male & & \\
Ethnicity & 1 & 10.0 \\
American Indian/Alaskan Native & 5 & 50.0 \\
Black/African American & 2 & 20.0 \\
Hispanic/Latino & 1 & 10.0 \\
Multi-Racial & 1 & 10.0 \\
White &
\end{tabular}

Note. $\mathrm{n}=10$ for ethnicity because of missing data

Table 2. Descriptive Statistics for Sample Continuous Demographics

\begin{tabular}{cccccc}
\hline Variable & $\mathrm{n}$ & Min. & Max. & M & SD \\
\hline Age & 11 & 24 & 59 & 49.55 & 10.62 \\
Years Education & 10 & 9 & 14 & 12.00 & 1.63 \\
\hline
\end{tabular}

Note. $\mathrm{n}=10$ for education because of missing data

Table 3. Descriptive Statistics for Condom \& Street Drug Use

\begin{tabular}{lcc}
\hline \multicolumn{1}{c}{ In the past month... } & N & $\%$ \\
\hline Used Condom During Each Sexual Activity & & \\
Yes & 5 & 45.5 \\
No & 6 & 54.5 \\
Used Street Drugs to Cope & & \\
Yes & 1 & 9.1 \\
No & 10 & 90.9 \\
\hline
\end{tabular}

Table 4. Frequencies \& Percents for Pre-Test Impact of Event Scale Items

\begin{tabular}{|c|c|c|c|c|c|c|}
\hline Item & & 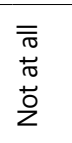 & 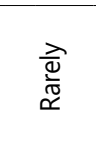 & & 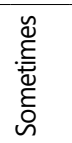 & 离 \\
\hline & $\mathrm{N}$ & \% & $\mathrm{N} \%$ & $\mathrm{n}$ & $\%$ & $\mathrm{~N} \%$ \\
\hline I thought about it when I didn't mean to. & 1 & 9.1 & 436.4 & & 18.2 & 436.4 \\
\hline $\begin{array}{l}\text { I avoided letting myself get upset when I } \\
\text { thought about it or was reminded of it. }\end{array}$ & 1 & 9.1 & 436.4 & & 36.4 & 218.2 \\
\hline I tried to remove it from memory. & 1 & 9.1 & 19.1 & & 18.2 & 763.6 \\
\hline $\begin{array}{l}\text { I had trouble falling asleep or staying asleep, } \\
\text { because of pictures or thoughts about it that } \\
\text { came into my mind. }\end{array}$ & 3 & 27.3 & 218.2 & 21 & 9.1 & 545.5 \\
\hline I had waves of strong feelings about it. & 1 & 9.1 & 436.4 & & 27.3 & 327.3 \\
\hline I had dreams about it. & 2 & 18.2 & 218.2 & & 27.3 & 327.3 \\
\hline I stayed away from reminders of it. & 1 & 9.1 & 19.1 & & 27.3 & 654.5 \\
\hline I felt as if it hadn't happened or it wasn't real. & 1 & 9.1 & 19.1 & & 36.4 & 545.5 \\
\hline I tried not to talk about it. & 1 & 9.1 & 19.1 & & 27.3 & 654.5 \\
\hline Pictures about it popped into my mind. & 1 & 9.1 & 218.2 & & 45.5 & 327.3 \\
\hline Other things kept making me think about it. & 0 & 0.0 & 327.3 & & 36.4 & 436.4 \\
\hline $\begin{array}{l}\text { I was aware that I still had a lot of feelings } \\
\text { about it, but I didn't deal with them. }\end{array}$ & & 10.0 & 3 & & 10.0 & 550.0 \\
\hline I tried not to think about it. & 1 & 9.1 & 19.1 & & 9.1 & 872.7 \\
\hline Any reminder brought back feelings about it. & 1 & 9.1 & 19.1 & & 36.4 & 545.5 \\
\hline
\end{tabular}


My feelings about it were kind of numb.

$\begin{array}{llllllll}0 & 0.0 & 3 & 30.0 & 5 & 50.0 & 2 & 20.0\end{array}$

Note. $\mathrm{N}$ varies because of missing data. Valid percentages are displayed.

Table 5. Frequencies \& Percents for Post-test Impact of Event Scale Items

\begin{tabular}{|c|c|c|c|c|}
\hline Item & 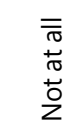 & $\frac{\vec{d}}{\frac{d}{\overparen{\varpi}}}$ & 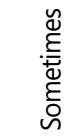 & $\stackrel{\frac{c}{\oplus}}{\stackrel{ \pm}{0}}$ \\
\hline & $\mathrm{N} \%$ & $\mathrm{~N} \%$ & n \% & $\mathrm{N} \%$ \\
\hline
\end{tabular}

I thought about it when I didn't mean to.

I avoided letting myself get upset when I

thought about it or was reminded of it.

327.3327 .3545 .500 .0

I tried to remove it from memory.

220.0330 .0440 .0110 .0

220.0330 .0440 .0110 .0

I had trouble falling asleep or staying asleep,

because of pictures or thoughts about it that $\begin{array}{lllllll}3 & 33.3 & 3 & 33.3 & 1 & 11.1222 .2\end{array}$

came into my mind.

I had waves of strong feelings about it.

I had dreams about it.

327.3436 .4436 .400 .0

I stayed away from reminders of it.

$2 \begin{array}{llllllll}2 & 18.2 & 5 & 45.5 & 4 & 36.4 & 0 & 0.0\end{array}$

220.0330 .0220 .0330 .0

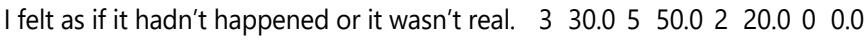

I tried not to talk about it.

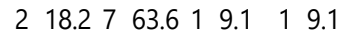

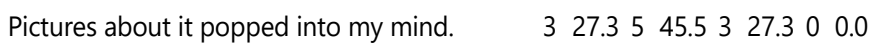

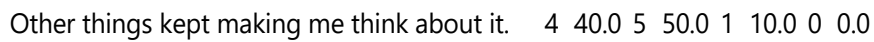

I was aware that I still had a lot of feelings

about it, but I didn't deal with them.

$436.4763 .600 .0 \quad 0 \quad 0.0$

I tried not to think about it.

$\begin{array}{llllllll}3 & 27.3 & 3 & 27.3 & 4 & 36.4 & 1 & 9.1\end{array}$

Any reminder brought back feelings about it. $\begin{array}{llllllll}2 & 18.2 & 6 & 54.5 & 3 & 27.3 & 0 & 0.0\end{array}$

My feelings about it were kind of numb.

$\begin{array}{llllllll}3 & 27.3 & 4 & 36.4 & 3 & 27.3 & 1 & 9.1\end{array}$

Note. $\mathrm{n}$ varies because of missing data. Valid percentages are displayed

Table 6. Wilcoxon Test

\begin{tabular}{|c|c|c|c|c|c|c|}
\hline Item & 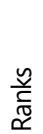 & $z$ & 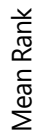 & 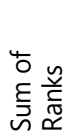 & $N$ & i무 \\
\hline
\end{tabular}

I thought about it when I didn't mean Negative Ranks $4 \quad 4.2517 .00$-1.38.167

to. Positive Ranks 22.004 .00

I avoided letting myself get upset when Negative Ranks $5 \quad 4.2021 .00$ I thought about it or was reminded of

Positive Ranks $35.0015 .00^{-0.43 .667}$

I tried to remove it from memory.

Negative Ranks $7 \quad 4.0028 .00$

Positive Ranks $0 \quad 0.000 .00$

$-2.41 .016$

I had trouble falling asleep or staying Negative Ranks $4 \quad 3.5014 .00$

asleep, because of pictures or thoughts

about it that came into my mind.

Positive Ranks 11.001 .00

$-1.75 .080$

I had waves of strong feelings about it. Negative Ranks 53.7018 .50

Positive Ranks 12.502 .50

$-1.69 .092$

Negative Ranks $5 \quad 4.6023 .00$

Positive Ranks 22.505 .00

$-1.53 .125$

I had dreams about it.

Negative Ranks $6 \quad 4.0824 .50$

Positive Ranks 13.503 .50

Negative Ranks $8 \quad 4.5036 .00$

Positive Ranks $0 \quad 0.000 .00$

Negative Ranks 84.5036 .00

Positive Ranks $0 \quad 0.000 .00$

Negative Ranks $6 \quad 4.3326 .00$

Positive Ranks 12.002 .00

Negative Ranks 105.5055 .00

Positive Ranks $0 \quad 0.000 .00$

Negative Ranks $7 \quad 5.0035 .00$

Positive Ranks 11.001 .00

$-2.40 .017$

feelings about it, but I didn't deal with them.

I tried not to think about it.

Negative Ranks $8 \quad 4.5036 .00$

Positive Ranks $0 \quad 0.000 .00$
Any reminder brought back feelings about it.

My feelings about it were kind of numb.

Negative Ranks 95.6751 .00

Positive Ranks 14.004 .00

Negative Ranks 64.5827 .50

Positive Ranks $24.258 .50-134.180$

(.)

Table 7. Descriptive Statistics Summary

\begin{tabular}{llll}
\hline Variable & $\mathrm{n}$ & $\mathrm{M}$ & $\mathrm{SD}$ \\
\hline Overall Pre-test & 11 & 3.13 & 1.33 \\
Overall Post-test & 11 & 0.91 & 0.27 \\
\hline
\end{tabular}

Table 8. Paired t-test Statistics

\begin{tabular}{llllllll}
\hline Paired Differences \\
\hline Mean & $\begin{array}{l}\text { Std. } \\
\text { Deviation }\end{array}$ & $\begin{array}{l}\text { SE of } \\
\text { Mean }\end{array}$ & \multicolumn{9}{c}{$95 \%$ Cl of the Difference } & T & df & Sig \\
\hline 1.61 & 1.44 & 0.43 & 0.64 & 2.57 & 3.72 & 10 & .004 \\
\hline
\end{tabular}

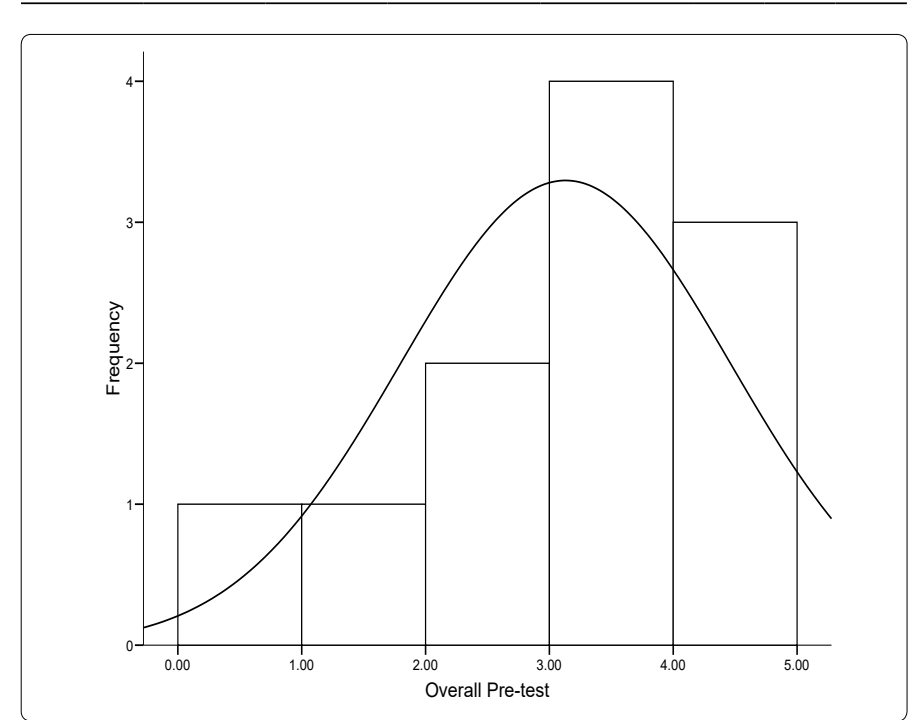

Figure 1. Distribution of Pre-Test Impact of Event Scores

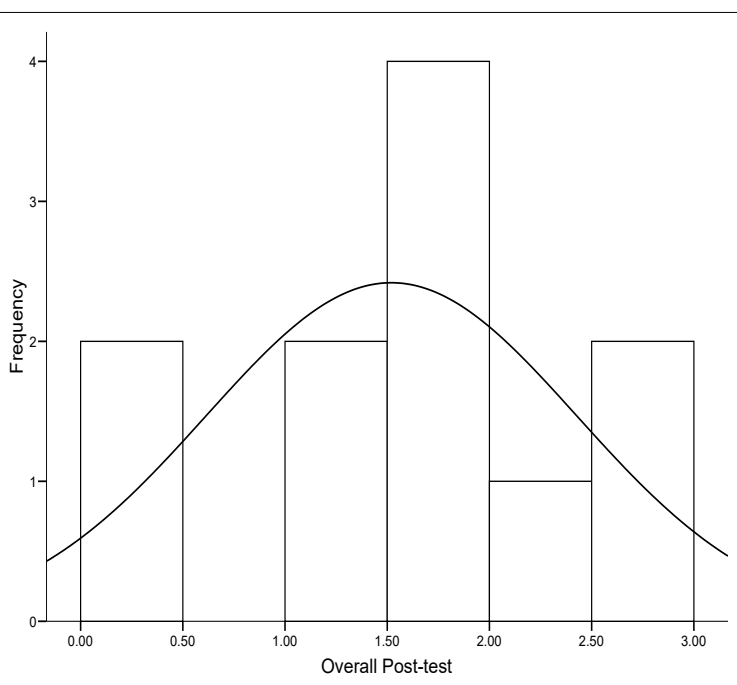

Figure 2. Distribution of Post-Test Impact of Event Scores

\section{Analysis of Data Outcomes}

A quantitative quasi experimental one group design was used to obtain the information; whether did the 90 minute, 8 weekly group cognitive behavioral therapy (CBT) interventions reduce traumatic stress and HIV transmission behaviors. The IBM Statistical Package for Social Science (SPSS Statistics), Version 19used for data analysis. Wilcoxon T Tests were used to assess differences in pre-intervention and post-intervention IES scores. A confidence interval of $95 \%$ was used with alpha set at 
0.05 [28]. From a descriptive standpoint it was clear that the participants' were more likely to respond 'often' or 'sometimes' to the thoughts and behaviors measured by the scale at pretest compared to the post-test. For example, the most common responses for 11 of the 15 items were 'often' at pre-test, but 'often' was the most common response in only 1 of 15 items at the post-test. The same descriptive pattern was revealed for the 'not at all' responses. At pre-test the 'not at all' responses were most common for 0 of the 15 items. The author analyzed data to determine which of the items significantly changed from pre-test to post-test. Several (one for each item) were conducted to determine if there were significant changes in the scale items from pre-test to post-test. The Wilcoxon test is the non-parametric (i.e., assumption and distribution-free) equivalent of the paired-samples t-test. It is appropriate when comparing two related (i.e., paired) ordinal variables. It was used in this case because each test was conducted on an individual ordinal scale item from the Impact of Event Scale.

The Wilcoxon test [28] statistics for each item are listed in table 6. The Wilcoxon tests revealed significant decreases from pre-test to post-test in the frequency of negative thoughts and behaviors for 8 of the 15 items. The significant differences are in bold. For example, there was a significant decrease from pretest to post-test for the item 'I tried to remove it from memory,' $z=-2.41, p=.016$. The largest decrease from pre-test to posttest was for the item 'Other things kept making me think about it, $z=-2.82, p=.005$. These decreases from pre-test to posttest indicated that the participants' were thinking more positively and coping with their traumatic events for a majority of the items. The non-significant results should be interpreted with caution given the small sample size.

A paired-samples t-test was conducted to determine if there was a significant change from pre-test to post-test for the participants' overall responses to the Impact of Event Scale. The paired t-test was appropriate when assessing two related (i.e., paired) samples from pre-test to post-test. The mean was calculated for each item among the participants who responded, and this value replaced any missing responses for each question. Thus, all 11 participants were retained in the t-test analysis. A mean composite score of the 15 items was calculated to represent the participants' overall score.

\section{Summary of Findings}

The findings affirmed that CBT group interventions are effective behavioral group interventions to reduce traumatic stress and HIV transmission behaviors. The findings are consistent with the results of randomized clinical trials [9,23]. Consequently, the evidence support practice change project and the use of CBT group prevention/intervention targeted at HIV-positive women with histories to reduce stress and HIV transmission behaviors.

\section{Discussion}

The main findings in the study are categorized into adaptive and maladaptive coping behaviors. Adaptive coping behaviors included: trust, social engagement, effective communication, self-respect, supportive healthy relationships, and increased church attendance. Other positive coping behaviors were journal writing, reflective activities, condom negotiation skills, self-care, effective emotion focused and problem focused skills, post-traumatic growth, and adherence with HIV treatments, and financial management.

Conversely, major maladaptive behaviors that emerged during the study were: anger, lack of trust, avoidance, intrusive thoughts, high-risk sexual risk behaviors, diminished quality of life, and drug use. Moreover, a few women reported engaging in unprotected sex, dysfunctional relationships, and non-adherence with HIV medications to cope with shame, guilt, feelings of aloneness, and traumatic stress. These maladaptive behaviors were significantly reduced at the completion of the eight weekly group sessions. Thus, the results confirmed the scholarly inquiry question.

Data indicated that approximately half $(n=6,54.5 \%$ of the participants had not used condoms during the past month. Similarly, Carvalho et al. [19] found through a systematic review that behavioral interventions were not effective in promoting condom use among HIV positive women. One should use caution interpreting the participants' responses about not using condoms during the past month at baseline and post-intervention. The absence of condom use does not necessarily mean the participants did not use protective barriers. In addition, a large majority $(n=10,90 \%$ of the participants indicated they had not used street drugs to cope within the past month.

Participants were asked to respond to the 15-item (IES) [20]. The scale was designed to assess the frequency of thoughts and behaviors related sexual traumatic experiences during the past month. Outcome data indicated that participants were more likely to respond 'often' or 'sometimes' to the thoughts and behaviors measured by the scale at pre-test compared to posttest. For example the most common responses for 11 of the 15 items were 'often at pre-test, but often was the most common response in only 1 of 15 items at the post- test. In addition, the results revealed significant decreases in post-intervention group scores in the following statements:

- I tried to remove it from memory.

- I felt as if it hadn't happened or it wasn't real.

- I tried not to talk about it.

- Pictures about it popped into my mind.

- Other things kept me from making think about it.

- I was aware that I still had a lot of feelings about it, but I didn't deal with them.

- I tried not to think about it.

- Any reminder brought back feelings about it.

This suggests that the women made connections between sexual trauma experiences and avoidant coping behaviors such as substance abuse, risky sexual behaviors, and relationship patterns were powerful components of the CBT group interventions. These connections enhanced problem focused and emotion focused increasing motivation and group participation. In addition as the group progressed, women spoke openly about experiencing shame, anger, and 
guilt feelings associated with childhood sexual abuse. In one group session, participants compared feelings about CSA with HIV infection. They seemed shocked knowing their feelings were similar.

\section{Limitations}

Small sample size is a major limitation. Additionally, experts recommended 15 weekly, 90 minute group sessions. Further, high level control studies investigating the influence of CSA among HIV positive in developing countries are indicated. Despite these limitations, this study has significant implications for research, policy, and translation into clinical practice.

\section{Conclusion}

Human immunodeficiency virus is a chronic, but life threatening disease. Research is needed to design evidencedbased protocols to treatment and evaluate behavioral outcomes of HIV-positive women with histories of CSA. Research is also needed to understand the complexities associated with CSA, drug use, and sexual risk behaviors. As such, as the health care field moves towards evidence-based practice, it becomes ever more critical for health care providers to conduct systematic reviews of research literature for guiding programmatic activities, policy-making decisions, and future research in developed and undeveloped countries.

\section{References}

1. UNAIDS Joint United Nations Programme on HIV/AIDS. Women and girls and HIV. www.unaids.org. Accessed June 13, 2018.

2. UN Women. Hidden in plain sight: A statistical analysis of violence against children. UNICEF. 2018.

3. Chin D, Myers HF, Zhang M. Who improved in a trauma intervention for HIV-positive women with child sexual abuse histories? Psycho Trauma. 2014; 6(2): 152-58. doi: 10.1037/a0032180

4. Meade CS, Drabkin AS, Hansen NB, et al. Reductions in alcohol and cocaine use following a group coping intervention for HIV-positive adults with childhood sexual abuse histories. Addiction. 2010; 105(11): 1942-51. doi: 10.1111/j.1360-0443.20.03075x

5. Manglio R. The impact of childhood on health: A systematic review of reviews. Clinical Psychology. 2009; 29(7): 647-657. doi: 10.1016/j.cpr.2009.08.003

6. Whetten HW, Wilson CS. An examination of risky sexual behavior and HIV in victims of child abuse and neglect: A 30-year follow-up. Health Psychol. 2008; 27: 149-158. doi: 10.1037/0278-6133.27.2.149

7. Wyatt $\mathrm{GE}$, Longshore $\mathrm{D}$, Chin $\mathrm{D}$. The efficacy of an integrated risk reduction intervention for HIV positive women with child sexual abuse histories. AIDS and Behav. 2004; 8(4): 453-462. doi: 10.007/s10461-004-7329-y

8. Lloyd S, Operario D. HIV risk among men who have sex with men who have experienced childhood sexual abuse: Systematic review and metaanalysis. AIDS Edu Prev. 2012; 24(3): 228-41. doi: 10.1521/aeap.2012.24.3.228

9. Sikkema KJ, Hansen NB, Kochman A. Outcomes from a group intervention for coping with HIV/AIDS and childhood sexual abuse: Reductions in traumatic stress. AIDS Behav. 2007; 11(1): 49-60 doi: 10.1007/s1046-006-
$9149-8$

10. Sikkema KJ, Wilson PA, Kochman A, et al. Effects of a coping intervention on transmission risk behavior among people living with HIV/AIDS and a history of childhood sexual abuse. J Acquir Immune Defic Syndr. 2008; 47(4): 506-513. doi: 10.1097/QAl.0b013e318160d727

11. Sikkema KJ, Kochman A, van den Berg JJ, Hansen NB, Watt MH, et al. Living in the face of trauma: An intervention for coping with HIV and childhood sexual abuse. Duke University, Department of Psychology and Neuroscience. Accessed April 5, 2013.

12. Colangelo J, Keefe-Cooperman K. Understanding the Impact of Childhood Sexual Abuse on Women's Sexuality. Journal of Mental Health Counseling. 2012; 12: 34(1): 14-37. doi: 10.17744/mehc.34.1.e045658226542730

13. Wilson DR. Health consequences of childhood sexual abuse. Perspect in Psychiatr Care. 2010; 46(1): 56-64. doi: 10.1111/j.1744-6163.2009

14. Lazarus RS, Folkman S. Stress, Appraisal, and Coping. New York, NY: Springer; 1984.

15. Leserman J. Role of depression, stress, and trauma in HIV disease. Psychosom Med. 2008; 70: 539-545. doi:10.1097/PSY.0b013e3181777a5f

16. Leserman J, Pence GW, Whetten K. Relation of lifetime trauma and depressive symptoms to mortality in HIV. AmJ Psychiatry. 2007; 165(11): 1707-13. doi: 10.1176/appi.appi.ajp.2007.06111775

17. National Institute of Mental Health. HIV-positive Survivors of Sexual Abuse Who Receive Coping Intervention Less Likely to Engage in Unprotected Sex. Science Update. 2008.

18. Crepaz N, Marshall JK, Aupont LW. The efficacy of HIV/STI behavioral interventions for African-American females in the United States: A metaanalysis. Am J Public Health. 2009; 99(11): 2069-2078. doi: 102121005/ AJPH.2008. 139519

19. Carvalho FT, Tonantzin R, Faria ER. Behavioral interventions to promote condom use among women living with HIV. Cochrane Database Sys Rev. 2012; 7(9): CD007844. doi: 10.1002/14651858.CD007844.pub2

20. Horowitz M, Wilner N, Alvarez W. Impact of event scale: A measure of subjective stress. Psychosom Med 1979; 41: 209-218.

21. Wong $\mathrm{FL}$, Rother-Borus MJ, Lightfoot M. Effects of behavioral intervention on substance use among people living with HIV: The healthy living project randomized controlled study. Addiction. 2008; 103(7): 1206-14. doi: 10.11111/j.1360-0443.2008.02222.x

22. CDC. Health Behaviors of Adults: United States, 2008-2010. US Department of Health and Human Services.

23. Chesney MA, Chambers DB, Taylor JM, Johnson LM, Folknan S, et al. Coping effectiveness training for men living with HIV: Results from a randomized clinical trial testing group-based interventions. Psychom Med. 2003; 65(6): 1038-46.

24. Puffer E, Kochman A, Hansen NB, Sikkema KL. An evidenced based group intervention for women living with HIV and history of childhood sexual abuse. Int J Group Psychother. 2011; 61(1): 99-126. doi: 10.1521/ ijgp.2011.61.1.98

25. Cocoran K, Fischer J. Measures for Clinical Practice: A Sourcebook. 1994; 3(2): New York, NY: Free Press.

26. Nightingale VR, Sher TG, Hansen NB. The impact of receiving an HIV diagnosis and cognitive process on psychological distress and posttraumatic growth. J Trauma Stress. 2010; 23(4): 452-460. doi: 10.1002/jts.20554

27. Burns N, Grove S. The Practice of Nursing Research: Appraisal, Synthesis, and Generation of Evidence. Philadelphia, PA: W.B. Saunders Company; 2009.

28. Howell DC. Fundamental Statistics for the Behavioral Sciences. Belmont, CA: Thomson-Wadsworth; 2010. 